\title{
Authors' reply to the letter from Mohadeseh Sani and Erfan Ayubi on: "Reliability and validity of a Turkish version of the Global Pelvic Floor Bother Questionnaire"
}

\author{
Nuriye Özengin ${ }^{1} \cdot$ Handan Ankarali ${ }^{2}$
}

Published online: 15 August 2016

(C) The International Urogynecological Association 2016

Sir,

We thank Drs. Sani and Ayubi for their interest in our article [1]. The Global Pelvic Floor Bother Questionnaire (GPFBQ) is a survey instrument for assessing the presence of pelvic floor dysfunction and bother severity. We frequently use the GPFBQ in our academic studies and clinic. We have found that the GPFBQ is a practical and global survey instrument because it consists of a low number of questions, and its application is quick and not time consuming.

The statistical analysis of the validity and reliability of the GPFBQ was conducted by Prof. Dr. Handan Ankarali of Düzce University Biostatistics and Medical Informatics Department. First, confirmatory factor analysis was applied in this study seeking to determine whether the GPFBQ scale, that consists of nine items and is described as singledimensional in the literature, is also single-dimensional in the analysis of research data. As a result of this analysis, the root mean square of residuals (RMSR) value was found to be 0.1008 , and an expected mean value of the RMSR for an acceptable model of 0.0877 (Kelley's criterion) was estimated for a single dimension. The RMSR value was greater than Kelley's criterion and therefore it was decided that the model is not appropriate for a single dimension. Explanatory factor analysis was applied following this stage and four dimensions appropriate for the analysis of research data were obtained. In our manuscript and the printed article the term "confirmatory factor analysis" was used four times instead of the correct term "explanatory factor analysis". Moreover, although we performed confirmatory factor analysis, the result was not reported in the article.

We regret these errors and hope that we have been able to clarify the statistical methods used in our study.

\section{References}

1. Doğan H, Özengin N, Bakar Y, Duran B (2016) Reliability and validity of a Turkish version of the Global Pelvic Floor Bother Questionnaire. Int Urogynecol J. doi:10.1007/s00192-016-3014-8.

This reply refers to the comment available at doi: $10.1007 / \mathrm{s} 00192-016-$ 3113-6

Nuriye Özengin

ozenginnuriye@yahoo.com

1 School of Physical Therapy and Rehabilitation, Abant Izzet Baysal University, 14280 Bolu, Turkey

2 Department of Biostatistics and Medical Informatics, Düzce University, 81620 Düzce, Turkey 